\title{
Effectiveness of Enhanced Recovery After Surgery (ERAS) for patients undergoing hysterectomy: A systematic review and meta-analysis
}

\author{
Chengyi Ho ${ }^{1}$, Hui Xian $\mathrm{Oh}^{1}$, Zi An Shian Seah ${ }^{1}$, Jiemin Zhu ${ }^{2}$, and Hong-Gu HE ${ }^{1}$ \\ ${ }^{1}$ National University of Singapore \\ ${ }^{2}$ Xiamen University
}

July 29, 2021

\begin{abstract}
Background: There is a lack of systematic review exploring the effectiveness of Enhanced Recovery After Surgery (ERAS) in hysterectomy in promoting better recovery. Objectives: To synthesize the best available evidence of the effectiveness of ERAS intervention in promoting better recovery of shortened length of hospital stay (primary outcome), lower readmission, and complication rates (secondary outcomes) among patients undergoing hysterectomy due to benign conditions as compared to conventional perioperative care. Search Strategy: Seven electronic databases were searched from the date of inception to December 2020. Selection Criteria: Randomized controlled trials, cohort studies, or quasi-experimental studies published in English examining the effects of ERAS for women diagnosed with benign gynecologic diseases and underwent either abdominal or laparoscopic hysterectomy were included. Data Collection and Analysis: Two reviewers independently conducted database search, data extraction, and methodological quality assessment. Meta-analyses were performed for all outcomes. The overall quality of evidence was assessed using GRADE. Main Results: Nine studies were included in this review. Meta-analysis showed a statistically significant reduction in length of hospital stay $(\mathrm{SMD}=-0.76,95 \% \mathrm{CI}[-1.06,-0.46], \mathrm{Z}=4.72, \mathrm{p}<.00001$ ), readmission rate $(\mathrm{RR}=0.65,95 \% \mathrm{CI}[0.44-0.96] ; \mathrm{Z}=2.16, \mathrm{p}=.03)$ and complication rate $(\mathrm{RR}=0.61,95 \% \mathrm{CI}[0.48-0.77] ; \mathrm{Z}=$ $4.17, \mathrm{p}<.0001)$, with high certainty of evidence. Conclusions: The effectiveness of ERAS in improving recovery indicates that hospitals could adopt the protocol to improve patients' health and wellbeing. Future studies can focus more on standardizing the protocol's elements.
\end{abstract}

\section{Hosted file}

A- Final BJOG Manuscript_21 July 2021.docx available at https://authorea.com/users/428130/ articles/532105-effectiveness-of-enhanced-recovery-after-surgery-eras-for-patientsundergoing-hysterectomy-a-systematic-review-and-meta-analysis

\section{Hosted file}

A- Final BJOG Tables and figures_20 July 2021.docx available at https://authorea.com/ users/428130/articles/532105-effectiveness-of-enhanced-recovery-after-surgery-eras-forpatients-undergoing-hysterectomy-a-systematic-review-and-meta-analysis 